\title{
The effect of dietary fish oil-supplementation to healthy young men on oxidative burst measured by whole blood chemiluminescence
}

\author{
Stine Bartelt ${ }^{1,2}$, Michael Timm ${ }^{2}$, Camilla T. Damsgaard ${ }^{1}$, Erik W. Hansen ${ }^{2}$, Harald S. Hansen ${ }^{2}$ \\ and Lotte Lauritzen ${ }^{1 *}$ \\ ${ }^{1}$ Department of Human Nutrition, Faculty of Life Sciences, University of Copenhagen, Rolighedsvej 30, DK-1958 Frederiksberg C, \\ Denmark \\ ${ }^{2}$ Department of Pharmacology and Pharmacotherapy, Faculty of Pharmaceutical Sciences, University of Copenhagen, \\ Universitetsparken 2, DK-2100 Copenhagen, Denmark
}

(Received 12 June 2007 - Revised 30 August 2007 - Accepted 24 September 2007 - First published online 17 January 2008)

Dietary long-chain $n$-3 PUFA ( $n$-3 LCPUFA) are thought to have immune-modulating effects, but the specific effects and mechanisms are not fully elucidated. The aim of this study was to determine whether dietary $n-3$ LCPUFA could affect ex vivo oxidative burst in healthy young men. The study had a randomised $2 \times 2$-factorial design in which subjects were randomly assigned to 8 -week supplementation with capsules containing fish oil (about $2.9 \mathrm{~g} n$-3 LCPUFA/d) or olive oil (control). Subjects were also randomly assigned to household use of oils and fat spreads with a high or a low $18: 2 n-6$ content. At baseline and at the end of the intervention, the fatty acid composition of peripheral blood mononuclear cells (PBMC) was analysed by GLC and oxidative burst was studied in whole blood stimulated with zymosan using luminol-enhanced chemiluminescence. The PBMC content of $n$-3 LCPUFA was markedly increased by the fish oil-supplementation $(P<0 \cdot 001$, compared to the olive oil groups). No effect of the intervention was observed on neutrophil count, but one measure of the zymosan-induced oxidative burst was higher in the fish oil groups $(P=0.03)$ compared to the olive oil groups. The fat intervention did not in itself affect oxidative burst neither did it change the effect of the fishoil intervention. The measures of oxidative burst at the end of the intervention period were found to be associated with the DHA content of PBMC ( $r 0.44, P=0.016)$, suggesting a dose-response relationship. These results indicate that $n$-3 LCPUFA may have immuno-stimulating effects.

Respiratory burst: Dietary supplementation: Inflammation: Human intervention study

Dietary long-chain ( $\geq$ C20) $n$-3 PUFA ( $n$-3 LCPUFA) are generally considered to be immuno-suppressive ${ }^{(1)}$ and have been shown to favourably affect a number of inflammatory diseases including rheumatoid arthritis ${ }^{(2)}$ and $\mathrm{CVD}^{(3,4)}$. Neutrophils are central players in inflammatory diseases ${ }^{(5)}$ as they form part of the innate immune response, which acts in the early host defence. Neutrophils kill microbes by releasing proteolytic enzymes, superoxide and related reactive oxygen species (ROS), a process referred to as oxidative burst. ROS are toxic to the surrounding tissue, and the damage provoked intensifies neutrophil activation, recruits more neutrophils and thereby increases the inflammatory response $^{(6)}$.

Feeding fish oil (FO), which contains $n-3$ LCPUFA, primarily DHA and EPA, to laboratory animals has been shown to decrease ex vivo neutrophil superoxide production measured in isolated neutrophils ${ }^{(7,8)}$. A number of ex vivo studies have investigated the influence of FO supplements on oxidative burst in healthy human volunteers, but the results are conflicting. Some have found suppressive effects of $\mathrm{FO}^{(9-12)}$ whereas others have not ${ }^{(13-17)}$.

The mechanisms by which $n-3$ LCPUFA exert their immune-modulating actions are unknown. From a classical point of view, the immuno-suppressive effect is thought to operate through changes in eicosanoid production. It has, however, also been suggested that $n-3$ LCPUFA mediate their effect by changes in gene expression or membrane structure $^{(18,19)}$. The NADPH oxidase, which is responsible for oxidative burst, is a large membrane-associated protein complex that assembles in lipid rafts ${ }^{(20)}$. Lipid rafts are generated by separation in the membrane bilayer of cholesterol and phospholipids containing unsaturated fatty acids, especially $\mathrm{DHA}^{(21)}$. It is therefore plausible that the activity of the NADPH oxidase could be affected by incorporation of DHA into cellular membranes.

A common characteristic of many of the previous studies investigating the effects of $n-3$ LCPUFA on oxidative burst is that the oxidative burst assays were performed on isolated

Abbreviations: ANCOVA, analysis of covariance; AUC, area under the curve; Des, desensitisation; FA\%, fatty acid area percentage; FO, fish oil; $n$-3 LCPUFA, long-chain n-3 PUFA; OO, olive oil; PBMC, peripheral blood mononuclear cell; R/K, rapeseed oil/Kaergaarden; ROS, reactive oxygen species; S/B, sunflower oil/Becel; $\mathrm{T} \frac{1}{2} \mathrm{P}$, time to half peak height; $\alpha_{40-60 \%}$, slope between 40 and $60 \%$ of peak height; $\Delta$, changes in (endpoint value - baseline value).

* Corresponding author: Dr Lotte Lauritzen, fax + 45353324 83, email 11@life.ku.dk 
neutrophils ${ }^{(9,11-14,22)}$. Recently, whole blood assays have been developed ${ }^{(10,15,17)}$, but in most cases the blood is kept on ice prior to analysis (at $37^{\circ} \mathrm{C}$ ). Isolating, centrifuging, cooling and heating neutrophils may change lipid structure $^{(21,23)}$. Especially in membranes with an increased content of $n-3$ LCPUFA $^{(24-26)}$ as unsaturated fatty acids are structurally more temperature-sensitive than SFA ${ }^{(21)}$. This in turn could affect neutrophil function and NADPH oxidase activity $^{(20,27)}$.

In the present study we investigated whether 8 weeks of dietary intervention affected ex vivo neutrophil oxidative burst in healthy young men. In this $2 \times 2$-fractional intervention, FO supplements were combined with household use of fats and oils with a high or low $18: 2 n-6$ content. The effect of the intervention on oxidative burst was measured ex vivo using a whole blood chemiluminescence assay. The temperature was kept at $37^{\circ} \mathrm{C}$ in order to mimic a normo-physiological situation and avoid excessive manipulation of the neutrophils.

\section{Methods}

\section{Subjects and study design}

The present study was part of a larger intervention study investigating the effects of FO-supplementation in combination with a high or low $18: 2 n-6$ intake on tissue PUFA-incorporation ${ }^{(28)}$, CVD risk markers and cytokine production in healthy young men. The study ran from October 2005 to February 2006. Healthy male subjects aged 1840 years were recruited by notes at universities throughout Copenhagen. Subjects were invited to participate in the study if they matched the following inclusion criteria: BMI $18.5-27 \mathrm{~kg} / \mathrm{m}^{2}$; non-smokers or infrequent smokers ( $\leq 5$ cigarettes a week); daily consumption of butter, margarine and/or oil, and home-made cooking $\geq 5$ times per week. Volunteers were excluded if they suffered from chronic diseases or serious allergic symptoms, were taking medication or dietary supplements, exercised more than $7 \mathrm{~h}$ weekly or had donated blood within the last 2 months. Ethical permission for the study was obtained from the Ethical Committee of the Municipalities of Frederiksberg and Copenhagen (Journal No. KF 01 267804). The study was registered in the clinical trial database of the US National Institutes of Health (ClinicalTrials.gov, NCT00266292). Informed written consent was obtained from all subjects enrolled in the study.

The design of the study was as follows: a screening visit, a 2-week run-in period, a baseline visit, an 8-week intervention period, an endpoint visit and a washout visit 8 weeks postintervention $^{(28)}$. The sub-study described in the present paper ended after the subjects had completed the endpoint visit and therefore does not contain any washout data. The following sections describe only measurements relevant to this particular sub-study.

The study had a randomised-parallel $2 \times 2$-factorial design in which the subjects were randomly assigned to daily supplementation with capsules containing either FO or olive oil (OO, control) for 8 weeks. Subjects were also randomly assigned to household use of oil and fat spreads with either a high or a low $18: 2 n-6$ content supplied by sunflower oil/ Becel (S/B) and rapeseed oil/Kaergaarden (R/K), respectively.
The randomisations were performed in two steps: first, notes were drawn from one envelope for the capsule intervention and second, within each capsule group notes were drawn for the oil and fat spread intervention. Thus, the subjects were randomly allocated in a double-blind fashion to one of four intervention groups: (1) OO-capsules and S/B, (2) OO-capsules and R/K, (3) FO-capsules and S/B or (4) FO-capsules and $\mathrm{R} / \mathrm{K}$.

Capsules (in a defined surplus), fat spreads and oils specified by the randomisation were supplied to the subjects on the day of their baseline visit. The subjects were instructed to consume ten capsules per day (equal to $5 \mathrm{ml} / \mathrm{d}$ ) with $\mathrm{FO}$ (Bio-Marin) or OO (both kindly provided by Pharma Nord, Vejle, Denmark). Each FO-capsule contained $333 \mathrm{mg} n-3$ PUFA (NEFA, approximately $166 \mathrm{mg}$ EPA and $119 \mathrm{mg}$ DHA). The OO was given as TAG, but the capsules were matched for fat content. The S/B groups were supplied with sunflower oil (kindly provided by Aarhus United Denmark A/S, Aarhus, Denmark) and margarine (Becel 60, kindly provided by Unilever Denmark A/S Foods, Brøndby, Denmark) with a high content of $18: 2 n-6$, whereas the $\mathrm{R} / \mathrm{K}$ groups were supplied with rapeseed oil (also a gift from Aarhus United Denmark A/S) and a butter product containing two-thirds butter and one-third rapeseed oil (Kaergaarden Light, kindly provided by Arla Foods amba, Viby, Denmark) with a low content of $18: 2 n-6$. Both the margarine and the butter products contained $60 \mathrm{~g}$ fat $/ 100 \mathrm{~g}$ product. Prior to the intervention period, all subjects were provided with butter (Lurpak, also a gift from Arla Foods amba) and $\mathrm{OO}$ (Oleificio R.M. s.p.a., Lucca, Italy) for a 2-week run-in period. The purpose of this was to reset the fatty acid composition of the tissue marker. There were no restrictions concerning the amount of fat spreads or oil ingested, but it was intended that the intake of fat and energy remained constant during the study. The average energy intake and the dietary composition were determined by $4 \mathrm{~d}$ weighed dietary records: (1) prior to the run-in period and (2) at the end of the 8-week intervention period. The mean length of the intervention was $56 \mathrm{~d}$ (range 50-65d).

Seventy-five subjects were invited to a screening visit at which height, weight, hip- and waist-circumference were measured and the subjects were instructed in how to perform the dietary records. Eight subjects dropped out during the run-in period due to the workload of attendance and one subject was rejected at the baseline visit due to intense discomfort during blood sampling. This left us with sixty-six men enrolled in the study, of which sixty-four completed the intervention period. The reasons for drop-out were discomfort swallowing the oil capsules $(n 1)$ and the workload of attendance $(n 1)$. Furthermore, six subjects were left out from this sub-study, four because no endpoint oxidative bursts were measured and two because the baseline neutrophil count failed (equipment error). Drop-outs and data collection failures were evenly distributed across the four intervention groups. Data are reported only for those subjects with a complete dataset ( $n$ 58). Furthermore, one analysis run stopped (for an unknown reason), which resulted in a loss of three subjects in all statistical tests of the area under the curve (AUC) and desensitisation (Des) oxidative burst variables ( $n$ 55). The baseline characteristics of the subjects in 
Table 1. Baseline characteristics of the subjects in the four intervention groups $(n 58)$

\begin{tabular}{|c|c|c|c|c|c|}
\hline & \multicolumn{2}{|c|}{ Olive oil capsules } & \multicolumn{2}{|c|}{ Fish oil capsules } & \multirow[b]{2}{*}{$P \dagger$} \\
\hline & $\mathrm{S} / \mathrm{B}(n 14)$ & $\mathrm{R} / \mathrm{K}(n 17)$ & $\mathrm{S} / \mathrm{B}(n 15)$ & $\mathrm{R} / \mathrm{K}(n 12)$ & \\
\hline Age (years) & & & & & 0.61 \\
\hline Median & 24 & 26 & 25 & 24 & \\
\hline Percentilesł & $22-27$ & $22-28$ & $22-27$ & $22-25$ & \\
\hline Height (m) & & & & & 0.17 \\
\hline Mean & 1.80 & 1.84 & 1.83 & 1.85 & \\
\hline SD & 0.06 & 0.06 & 0.07 & 0.08 & \\
\hline Weight (kg) & & & & & 0.36 \\
\hline Median & 74.4 & 79.4 & $72 \cdot 9$ & $75 \cdot 2$ & \\
\hline Percentiles & $69 \cdot 6-79 \cdot 0$ & $73 \cdot 1-84 \cdot 3$ & $65 \cdot 8-81 \cdot 3$ & $69 \cdot 3-82 \cdot 0$ & \\
\hline BMI $\left(\mathrm{kg} / \mathrm{m}^{2}\right)$ & & & & & 0.16 \\
\hline Mean & $23 \cdot 1$ & $23 \cdot 3$ & $22 \cdot 1$ & $22 \cdot 8$ & \\
\hline $\mathrm{SD}$ & 1.9 & 1.9 & 1.6 & $2 \cdot 2$ & \\
\hline Waist/hip ratio & & & & & $>0.99$ \\
\hline Median & 0.85 & 0.84 & 0.85 & 0.84 & \\
\hline Percentiles & $0.81-0.89$ & $0.83-0.87$ & $0.83-0.86$ & $0.83-0.88$ & \\
\hline
\end{tabular}

$\mathrm{R} / \mathrm{K}$, rapeseed oil/Kaergaarden; S/B, sunflower oil/Becel.

†Between-group comparisons were performed using one-way ANOVA and Kruskal-Wallis tests with significance established at $P<0.05$. No significant differences were observed for any of the variables. $\ddagger$ Percentiles: 25 th to 75 th.

each of the intervention groups are shown in Table 1. Age, height, weight, BMI and waist/hip ratio did not differ between the groups.

Fasting blood samples were collected at the baseline and endpoint visits. The subjects were asked to follow standardised fasting conditions before each visit. These were: no food for $12 \mathrm{~h}$ or more (except from 0.5 litre water), lack of strenuous physical activity for $36 \mathrm{~h}$, no smoking for 1 week and no medicine for $24 \mathrm{~h}$. Moreover, the subjects were told to eat the same meal the evening before all visits and to reschedule their visit if they were ill or had a cold. These criteria were checked at every visit.

\section{Experimental procedures}

On the days of examination, body weight and waist/hip ratio were determined. The subject filled out a questionnaire on dietary habits, illnesses, use of medication and smoking. Blood $(80-110 \mathrm{ml})$ was drawn from fasting males after $10 \mathrm{~min}$ of rest and analysed for: oxidative burst, cell count, $\mathrm{Hb}$ concentration and the fatty acid composition of peripheral blood mononuclear cells (PBMC).

Compliance was assessed by counting returned capsules and measuring tissue fatty acid composition of the PBMC. The mean daily consumption of capsule oils was 4.5 (SD 0.5 ) $\mathrm{ml} / \mathrm{d}$ (range $3 \cdot 0-5.5 \mathrm{ml} / \mathrm{d}$ ), equivalent to $89 \%$ compliance. No difference in compliance was observed between the intervention groups. The fatty acid composition in the total lipid fraction from PBMC was determined as a biomarker of the resulting changes in tissue composition.

\section{Measuring fatty acid composition of the tissue}

PBMC were isolated from blood sampled with Na-heparin and fatty acid composition was determined by GLC as described elsewhere ${ }^{(28)}$. Fatty acids are given as area percentage (FA\%), comparable to $\mathrm{g} / 100 \mathrm{~g}$.

\section{Neutrophil counts and Hb concentration}

Neutrophil count was determined by leucocyte size differentiation carried out on a Sysmex KX-21 automated haematology analyser (Sysmex Corporation, Kobe, Japan) on venous blood collected in EDTA tubes using the appropriate control. At the same time the $\mathrm{Hb}$ concentration was measured. Intra- and interassay $\mathrm{CV}$ for $\mathrm{Hb}$ were $0.7 \%$ (n 12) and $0.6 \%$ ( $n$ 25), respectively. Prior to the study intra-individual variation in neutrophil count was determined to $17.5 \%$ measured on three independent days in five individuals.

\section{Measurement of oxidative burst}

Prior to the study all stock solutions were portioned and frozen at $-20^{\circ} \mathrm{C}$. Zymosan A, a proteoglycan isolated from Saccharomyces cerevisiae (Sigma-Aldrich, St Louis, MO, USA), was suspended to $1 \mathrm{mg} / \mathrm{ml}$ in Hank's Balanced Salt Solution with $\mathrm{CaCl}_{2}+\mathrm{MgCl}_{2}$ (Invitrogen, Carlsbad, CA, USA) and portioned in aliquots of $300 \mu \mathrm{l}$, and luminol (5-amino-2,3-dihydro-1,4-phthalazinedione; Across Organics, NJ, USA) was dissolved to $5 \mathrm{mg} / \mathrm{ml}$ in dimethyl sulphoxide (Sigma-Aldrich) and portioned in aliquots of $100 \mu l$.

Blood, sampled in Na-heparin tubes for assessment of oxidative burst, was stored at $37^{\circ} \mathrm{C}$ and oxidative burst was determined within $2 \mathrm{~h}$ after sampling by chemiluminescence: $90 \mu \mathrm{l}$ whole blood diluted 1:9 in pre-warmed $\left(37^{\circ} \mathrm{C}\right)$ Hank's Balanced Salt Solution was loaded on ninety-six-well LumiNunc $^{\mathrm{TM}}$ polystyrene plates (Nunc, Roskilde, Denmark), which were preloaded with a 1:10 diluted stock solution of luminol $(10 \mu \mathrm{l})$ with Hank's Balanced Salt Solution in a final concentration of $142 \mu \mathrm{m} /$ well. The diluted blood samples were stimulated with $100 \mu \mathrm{l}$ pre-warmed $\left(37^{\circ} \mathrm{C}\right)$ zymosan at two different final concentrations (5 and $0.5 \mu \mathrm{g} / \mathrm{ml}$ ). Chemiluminescence (relative light units/s) was measured in a thermostat-regulated $\left(37^{\circ} \mathrm{C}\right)$ ninety-six-well Orion II Microplate 
luminometer (Berthold Detection Systems, Pforzheim, Germany). The microplate was read for $1 \mathrm{~s} /$ well with a 2 min delay, a cycle repeated for a total of seventy-five rounds. Unstimulated control samples (blanks) were analysed in duplicate and stimulated samples in sextuplicate.

Five variables were used to define the kinetics of the mean chemiluminescence response curve (Fig. 1): (1) time to half peak height $\left(\mathrm{T} \frac{1}{2} \mathrm{P}\right)$, (2) peak height, (3) AUC, (4) slope between 40 and $60 \%$ of peak height $\left(\alpha_{40-60 \%}\right)$ and (5) Des. All variables, except for AUC were calculated with Microsoft Excel 2000 (Windows XP; Microsoft Corp., Redmond, WA, USA) based on linear mathematical principles. AUC was determined with GraphPad Prism version 4.00 (GraphPad Prism, San Diego, CA, USA) based on integral calculation. Prior to the study, the inter-day variation of the oxidative burst variables was determined: blanks $2.4 \%$, peak height $25.9 \%$, AUC $29.6 \%$, T $\frac{1}{2} \mathrm{P} 3.8 \%$, Des $48.5 \%$ and $\alpha_{40-60 \%}$ $35.4 \%$ based on three independent days in five individuals. Data were analysed with the Statistical Package for the Social Sciences software version 13.0 (SPSS Inc., Chicago, IL, USA). Data are only shown for the $5 \mu \mathrm{g} / \mathrm{ml}$ zymosanstimulated samples. The results of the $0.5 \mu \mathrm{g} / \mathrm{ml}$ zymosanstimulated samples were similar, but the responses were smaller.

\section{Statistical analyses}

Data were checked for Gaussian distribution with ShapiroWilks test and visual inspection of histograms. Gaussian-distributed data are presented as means with their standard errors, whereas non-Gaussian-distributed data are presented as medians with 25 th -75 th percentiles. Baseline comparison between the four intervention groups were performed by one-way ANOVA and Kruskal-Wallis tests. Analyses of covariance (ANCOVA) were used for endpoint comparisons.

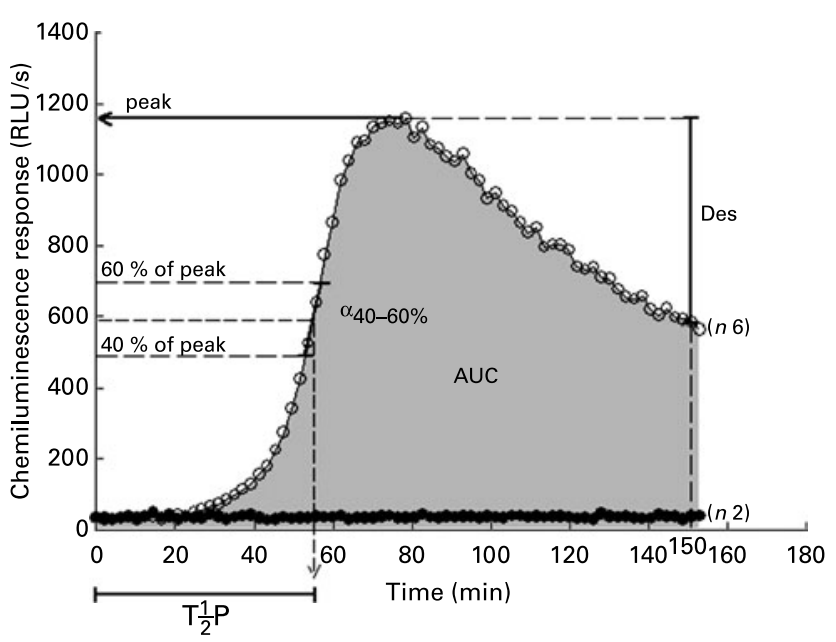

Fig. 1. Illustration of the luminol-enhanced chemiluminescence-detected oxidative burst response in whole blood as a function of time, based on a randomly selected participant. - Non-stimulated mean response curve; $\mathrm{O}$, mean zymosan (Saccharomyces cerevisiae)-induced $(5 \mu \mathrm{g} / \mathrm{ml}$ ) response curve. The response kinetics of the oxidative burst was expressed by five selected variables: peak height (peak); area under the curve (AUC, $\square$ ); time to half peak $\left(T \frac{1}{2} P\right)$; desensitisation (Des) and the slope between 40 and $60 \%$ of peak height $\left(\alpha_{40-60}\right)$. RLU, relative light units.
Non-Gaussian-distributed dependent variables (peak height, AUC, Des, $\alpha_{40-60 \%}$ ) were $\log (10)$-transformed before testing.

Statistical analysis was performed on the following: (1) the effect of the interventions on the fatty acid composition of PBMC, (2) the effect of the intervention on neutrophil count and oxidative burst, and (3) the association between the effect on oxidative burst and that on tissue fatty acid content. The three statistical procedures were as follows:

(1) The ANCOVA for the endpoint comparisons of PBMC fatty acid content (EPA, 22:5n-3, DHA and total $n-3$ PUFA) included fat type and capsule type as fixed factors and were adjusted for baseline values. All models were checked for interaction between the capsule and the oil/ fat spread interventions.

(2) All oxidative burst variables at endpoint were checked for bivariate Spearman's correlation with the following covariates: neutrophil count (endpoint), changes in $(\Delta$, endpoint value - baseline value) neutrophil count, smoking (yes/no) and $\Delta \mathrm{Hb}$ concentration. Correlating parameters (neutrophil count and $\Delta$ neutrophil count) were tested as covariates in the ANCOVA endpoint comparison of the oxidative burst variables, which also included fat type and capsule type as fixed factors (including check for fat $\times$ capsule interaction) and adjustment for baseline values. Covariates were successively removed, but those affecting the outcome $(P<0.05)$ were kept in the model $(\Delta$ neutrophil count).

(3) The dose-response relationship analysis of the oxidative burst variables were performed using multiple linear regression analysis including the following covariates: endpoint PBMC DHA content, baseline values and the covariates included in the oxidative burst variable ANCOVA.

Wilcoxon test and paired-sample $t$ test were used to check for within-group changes during the intervention of non-Gaussian-distributed and Gaussian-distributed data, respectively. Associations between the estimated FO-consumption (from the count of returned capsules $(\mathrm{ml} / \mathrm{d}))$ and $\Delta$ PBMC content of total $n-3$ PUFA, DHA, $22: 5 n-3$ and EPA was analysed using Pearson correlation analysis. Significance was established at $P<0.05$.

\section{Results}

The subjects had a mean energy intake of 12531 (SD 2742) kJ/d (range 6709-18 942 kJ/d), of which fat constituted 32.4 (SD 5.4) $\%$ (range 20.0-43.0\%) of energy. No between-group differences in energy consumption or macronutrient distribution were observed at baseline or during the intervention ${ }^{(28)}$. All subjects maintained a constant weight during the study.

\section{Fatty acid composition of mononuclear cells}

The effect of the interventions on PBMC fatty acid composition has been published recently ${ }^{(28)}$. In short, no differences were observed in the PBMC content of total $n-3$ PUFA, DHA, $22: 5 n-3$ or EPA between the treatment groups at baseline, after 2 weeks of run-in where oil and fat spreads were standardised. Endpoint comparison showed that 8 weeks of FO-supplementation increased total $n-3$ PUFA, DHA, $22: 5 n-3$ and 
EPA; all of which were significantly higher $(P<0.001$, for all $)$ in the FO groups. The effects were supported by pairedsample $t$-tests $(P<0.001$ for all fatty acid categories in both FO groups). The best FO compliance marker was EPA, which increased in the FO groups from 0.52 (SE 0.02) to 2.73 (SE 0.13) FA\% in the PBMC compared to no changes (0.44 (SE 0.02) to 0.39 (SE 0.02) FA\%) in the OO groups. The estimated FO-capsule consumption (count of returned FO-capsules $(\mathrm{ml} / \mathrm{d})$ ) correlated with endpoint PBMC EPA content $(r 0.51, P=0.006)$, total $n-3$ PUFA content $(r 0.48$, $P=0.011)$ and the DHA content $(r 0.38, P=0.049)$.

\section{Neutrophils}

The neutrophil counts at baseline did not differ between the four intervention groups. The median count was $2.4 \times 10^{9}$ cells $/ 1\left(25\right.$ th -75 th percentiles $2.0-3.2 \times 10^{9}$ cells $\left./ 1\right)$, ranging from 1.2 to $4.3 \times 10^{9}$ cells/l. The interventions had no effect on the cell counts. In general, the neutrophil count of each subject was relatively constant (mean change of $0.66(\mathrm{SD} 0.88) \times 10^{9}$ cells $\left./ \mathrm{l}\right)$, but in five of the subjects the cell count varied greatly $\left(\geq 1.8 \times 10^{9}\right.$ cells $\left./ 1\right)$ between the baseline and endpoint visit.

\section{Oxidative burst}

There were no differences between the four groups for any of the oxidative burst variables at baseline. Four of the five oxidative burst variables (with the exception of $\mathrm{T} \frac{1}{2} \mathrm{P}$ ) correlated with one another at baseline $(0.89<r<0.99, P<0.001)$ and after 8 weeks of intervention $(0.35<r<0.52, P \leq 0.007)$. Bivariate correlations were observed between four of the five oxidative burst endpoint variables (with the exception of $\mathrm{T} \frac{1}{2} \mathrm{P}$, $P=0.23)$ and endpoint neutrophil count $(0.35<r<0.52$, $P \leq 0.007)$. The oxidative burst endpoint variables were checked for correlation with confounders, such as $\mathrm{Hb}$ concentration (possible quenching of emitted light) and if the subject smoked (yes/no). No relations were observed, except for a correlation between $\Delta \mathrm{Hb}$ concentration and $\mathrm{T} \frac{1}{2} \mathrm{P}(r \quad 0 \cdot 29$, $P=0.03$ ).

Eight weeks of capsule intervention were found to have a significant effect on one of the five oxidative burst variables, Des $(P=0.03$; Table 2). No effects of the fat intervention or of the capsules $\times$ fat interaction term were observed (data not shown). Two outliers were observed in the ANCOVA model for peak height, AUC and Des (Fig. 2). After exclusion of these two subjects, Des $(P=0 \cdot 01)$, peak height $(P=0.02)$ and AUC $(P=0.05)$ were significantly increased in the FO-supplemented groups.

\section{Dose-response relationship}

Endpoint comparison of the oxidative burst variables correlated significantly with the DHA content in the PBMC at the end of the intervention ( $r 0 \cdot 44, P=0.016$ for Des and AUC shown in Fig. 3 ). As for the capsule effect on oxidative burst, the two subjects were also outliers in the dose-response relationship regression models (for peak height, AUC and Des). Exclusion of the two subjects in the analyses decreased the $P$ value for the dose-response relationships (Des $(P=0.010)$, peak height $(P=0.047)$ and AUC $(P=0.02))$. Similarly, a significant dose-response relationship was observed for EPA (Des $(P=0.029)$ and peak height $(P=0 \cdot 047))$, but to a lesser extent than the one observed for DHA.

\section{Discussion}

The present results indicate that FO may increase oxidative burst after 8 weeks of intervention, as one of five variables was significantly increased in relation to the control supplement. This was supported by outlier analysis and a doseresponse relationship between the oxidative burst and the DHA content of immune cells (here PBMC). The effect was most evident for the oxidative burst variables: peak height, AUC and Des, but the oxidative burst variables were highly inter-correlated. The present result is in agreement with a recent study that found enhanced oxidative burst in healthy young men after 8 weeks of FO-supplementation ${ }^{(29)}$. However, both these studies contradict the results from previous similar studies that have found either no effects ${ }^{(10,13-17)}$ or a reduction in the response $e^{(11,12)}$. The two early studies that observed a reduction in oxidative burst ${ }^{(11,12)}$ were small $(n 6$ and $n$ 22), had no control group, and gave little information on inclusion and exclusion criteria and participant characteristics (e.g. the mean age). In general, the more recent and larger studies ${ }^{(10,13-17)}$ have all reported no effect of $n-3$ LCPUFA-supplementation on oxidative burst in healthy young men. All of these, except one ${ }^{(13)}$, were randomised, placebo-controlled and double-blinded like the present study. The FO-dose used in the present study (about $2.9 \mathrm{~g} n$-3 LCPUFA/d) was within the range provided in the previous studies ${ }^{(10-12,14)}$. We had only minor drop-outs and compliance was good as judged from both capsule count and PBMC fatty acid analysis. In agreement with results from a previous study ${ }^{(17)}$, no effect was observed on neutrophil counts. Most of the subjects had very small changes in neutrophil count (comparable to the intra-individual variation measured in our pre-study), but a few varied more. Therefore, all analyses were adjusted for the changes in neutrophil count.

A study by Rees et al. $^{(10)}$ suggested that the effect of FOsupplementation may be age-related, since they found an immuno-suppressive effect on oxidative burst in a group of older subjects (aged 55-73 years), whereas no effect was observed in a group of younger subjects (aged 18-52 years). However, another study found no effect of FO-supplementation on oxidative burst in subjects aged 55-74 years $^{(17)}$. The most striking difference between these two studies was the type of oils that they used. Thies et al. ${ }^{(17)}$ used five different oils including a pure DHA oil $(700 \mathrm{mg} / \mathrm{d})$ and a FO that supplied $720 \mathrm{mg}$ EPA/d and $280 \mathrm{mg}$ DHA/d, whereas Rees et $a l^{(10)}$ used a pure EPA oil in four different doses. Thus, it might be of interest to investigate possible differences in the effects of EPA, DHA or a combination of the two on oxidative burst in elderly subjects. The opposing effects on the immune response in older versus young men might be related to health status. It has been shown that FO lowers cardiovascular risk markers, such as blood pressure ${ }^{(30)}$ and heart rate ${ }^{(31)}$, primarily in high-risk subjects, e.g. elderly or those with high baseline values. Whether the immunostimulating effect of FO in the present study has any longterm health effects is up for further investigation. Increased 
Table 2. Oxidative burst variables at baseline and endpoint in the four intervention groups $(n 58)$

\begin{tabular}{|c|c|c|c|c|c|c|c|c|c|c|}
\hline & \multicolumn{4}{|c|}{ Olive oil } & \multicolumn{4}{|c|}{ Fish oil } & \multirow[b]{3}{*}{ Fats $P$} & \multirow[b]{3}{*}{ Capsules $P$} \\
\hline & \multicolumn{2}{|c|}{$\mathrm{S} / \mathrm{B}(n 14)$} & \multicolumn{2}{|c|}{$\mathrm{R} / \mathrm{K}(n 17)$} & \multicolumn{2}{|c|}{$\mathrm{S} / \mathrm{B}\left(\begin{array}{ll}n & 15)\end{array}\right.$} & \multicolumn{2}{|c|}{$\mathrm{R} / \mathrm{K}(n 12)$} & & \\
\hline & Baseline & Endpoint & Baseline & Endpoint & Baseline & Endpoint & Baseline & Endpoint & & \\
\hline \multicolumn{11}{|l|}{ Blank (RLU/s) } \\
\hline Mean & 32 & 34 & 34 & 34 & 32 & 35 & 33 & 33 & 0.53 & 0.98 \\
\hline SD & 0.8 & 0.6 & 0.6 & 0.6 & 0.8 & 0.9 & 0.6 & 0.7 & & \\
\hline \multicolumn{11}{|l|}{ Peak (RLU/s) } \\
\hline Median & 1531 & $1104^{*}$ & 1961 & 1219 & 1500 & 1155 & 1345 & 1471 & 0.33 & 0.09 \\
\hline Percentiles $\dagger$ & $1417-2382$ & $857-2005$ & $1003-2637$ & $885-2802$ & $768-2194$ & $728-2059$ & $953-2198$ & $1101-1640$ & & \\
\hline \multicolumn{11}{|l|}{ AUC $\ddagger$} \\
\hline Median & 147285 & $96875^{\star}$ & 140861 & 106327 & 118235 & 100013 & 120713 & 124240 & 0.14 & 0.19 \\
\hline $\begin{array}{l}\text { Percentiles } \\
T_{11} P(\text { min) }\end{array}$ & \multirow{2}{*}{\multicolumn{5}{|c|}{$\mathrm{T} \frac{1}{2} \mathrm{P}(\mathrm{min})$}} & $65812-162256$ & $85314-207650$ & $100827-146135$ & & \\
\hline Mean & 52.48 & $52 \cdot 14$ & & & $52 \cdot 22$ & 52.43 & 52.61 & 52.11 & & \\
\hline SD & 0.52 & 0.53 & 0.72 & 0.54 & 0.62 & 0.91 & 0.71 & 0.36 & & \\
\hline \multicolumn{11}{|l|}{ Des $(R L U / s) \ddagger$} \\
\hline Median & 697 & $446^{*}$ & 654 & 493 & 447 & 538 & 438 & 541 & 0.12 & $0.03 \S$ \\
\hline Percentiles & $279-1026$ & $193-637$ & $310-1000$ & $284-912$ & $205-923$ & $277-918$ & $302-493$ & $363-809$ & & \\
\hline \multicolumn{11}{|c|}{$\alpha_{40-60}(\mathrm{RLU} / \mathrm{s}$ per min) } \\
\hline Median & $70 \cdot 9$ & $49 \cdot 8^{*}$ & 95.4 & $62 \cdot 0$ & $60 \cdot 1$ & 57.4 & $51 \cdot 7$ & 61.5 & $0 \cdot 10$ & 0.14 \\
\hline Percentiles & $56 \cdot 0-123 \cdot 0$ & $27 \cdot 6-89 \cdot 3$ & $34 \cdot 0-132 \cdot 4$ & $35 \cdot 9-136 \cdot 0$ & $27 \cdot 2-114 \cdot 3$ & $25 \cdot 0-103 \cdot 5$ & $36 \cdot 2-100 \cdot 6$ & $48 \cdot 0-81 \cdot 8$ & & \\
\hline
\end{tabular}

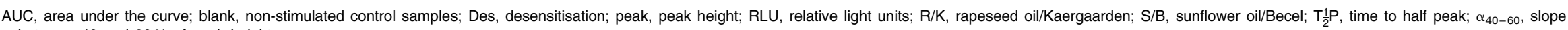
between 40 and $60 \%$ of peak height.

Values were significantly different from those of the baseline (paired-sample $t$-test or Wilcoxon test): peak, $P=0.016$; AUC, $P=0.016$; Des, $P=0.026 ; \alpha_{40}-60, P=0.013$.

†Percentiles: 25 th to 75 th.

$\S$ Endpoint between-group comparisons were performed using analysis of covariance with fats and capsules as fixed factors, adjusting for baseline values and changes in neutrophil count (endpoint - baseline). 
(A)

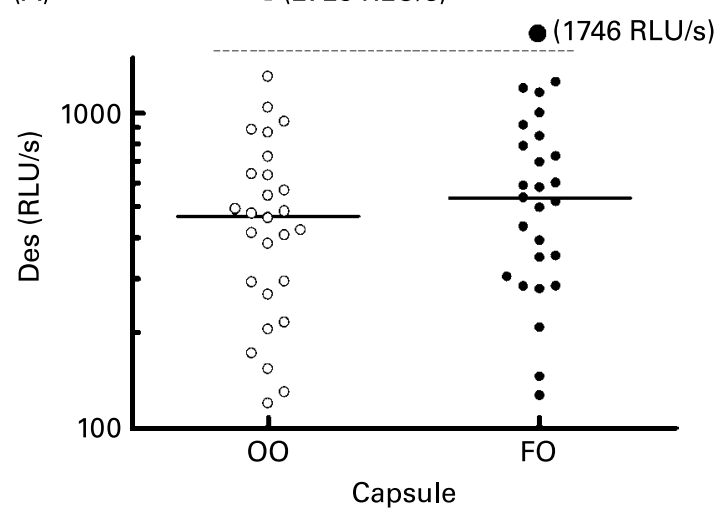

(B)

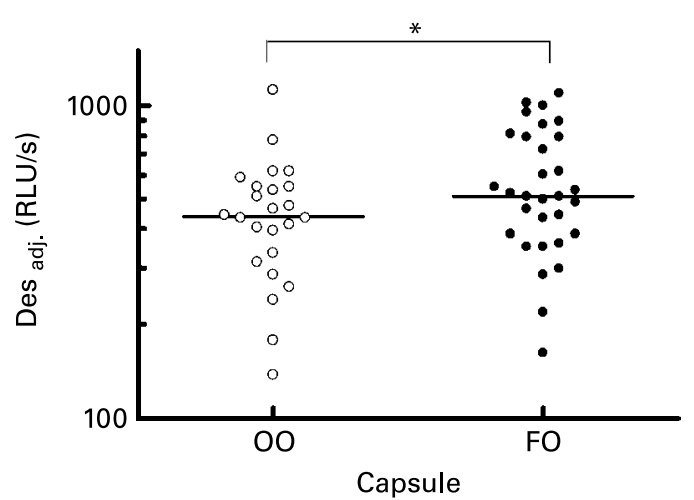

Fig. 2. The effect of the capsule intervention on desensitisation (Des). (A), Des values for each participant at the end of the intervention are plotted in each of the two capsule groups (OO, olive oil (control); FO, fish oil). Two subjects had outlying Des values. These are given above a break on the $y$-axis ( --$)$. (B), Des values were adjusted for baseline value and $\Delta$ neutrophil count. ${ }^{*}$ Significant difference between the oxidative burst in the capsule groups by analysis of covariance $(P=0.03)$. - Median; RLU, relative light units.

oxidative burst may have beneficial anti-bactericidal effects as shown in mice ${ }^{(32)}$.

The inconsistent results, concerning the effect of $n-3$ LCPUFA on oxidative burst, may to some extent be due to the method of assessment. The more recent studies have used fluorescence-activated cell-sorting analyses for detection of ROS in isolated neutrophils ${ }^{(29)}$ or whole blood ${ }^{(10,15,17)}$. The earlier studies have used isolated neutrophils assayed by either luminol-enhanced chemiluminescence ${ }^{(11,12,16)}$ or cytochrome $c$ reduction $^{(12-14)}$. The isolation procedure has a mechanical impact on the neutrophils and may change the structure and function of the cells. All the methods rely on chemical trapping of ROS, which due to the extreme reactivity of superoxide is rapidly converted to other ROS. Some ROS are able to cross membranes, which makes it difficult to distinguish between intracellularly and extracellularly produced ROS.

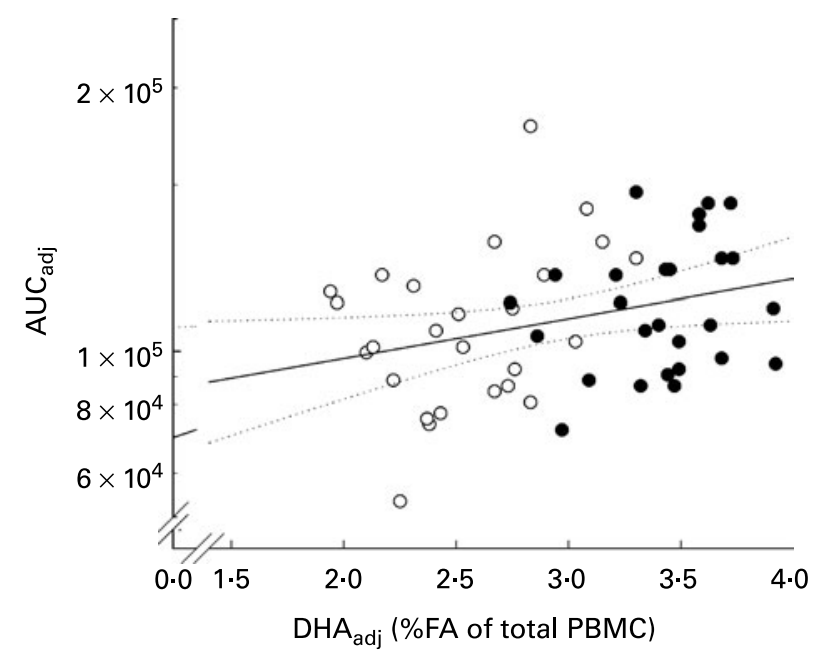

Fig. 3. The correlation between DHA in the peripheral blood mononuclear cells (PBMC) and the oxidative burst expressed as area under the curve (AUC) after 8 weeks of intervention with capsules with fish oil $(\bullet)$ or olive oil (control, O). AUC and PBMC DHA were adjusted for baseline value of oxidative burst and changes in neutrophil count during the intervention. Regression curve with $95 \% \mathrm{Cl}(\ldots)$ based on $n 55$ ( $r 0.27, P=0.04)$; \%FA, fatty acid area percentage.
Luminol-enhanced chemiluminiscence is thought to measure intracellular as well as extracellular ROS, whereas the fluorescence-activated cell-sorting analyses only detect the intracellular ROS. More methods could be used in parallel in order to enhance the validity of the results. To our knowledge, this is the first study to use chemiluminescence to study the effect of FO-supplementation on oxidative burst ex vivo in whole blood. The advantage of the whole blood assay is that the neutrophils are kept in their physiological environment. We handled the blood for the assay as gently as possible throughout the entire procedure and performed the assay at $37^{\circ} \mathrm{C}$. Furthermore, we used a sub-maximal stimulus (a zymosan-neutrophil ratio of 2.4) to induce an oxidative burst, which may also be more physiologically relevant than the use of surplus stimulus to ensure a $100 \%$ cell activation as is done in most other studies ${ }^{(10,15,17)}$.

We used OO as capsule control because it has a low content of PUFA, especially $n$-3 PUFA, and because $18: 1 n-9$ was regarded as a neutral fatty acid. The use of an OO control in studies of immune function has been questioned since some studies have reported effects on immune function after OO-supplementation ${ }^{(33,34)}$. The observed effect of the capsule intervention appears to be due to a reduction in the oxidative burst, especially when assessed as Des, in the OO groups rather than an increase in the FO groups. However, due to the observed correlation between the oxidative burst effect and the PBMC content of DHA we find it unlikely that the effect is driven by the OO. The observed dose-response correlation was not so strong $(r 0 \cdot 27, P=0 \cdot 04)$, but since this was interfered with by inter-day variation (biological as well as methodological, which for AUC amounted to 29.6\%) it may in reality represent a stronger true biological dose-response relationship. A dietary PUFA-induced increased membrane DHA content in Jurkat T-cells was shown to be paralleled by a decrease in MUFA ${ }^{(35)}$. No inverse association between DHA and MUFA was observed in the present study (data not shown) and it is therefore not likely that the correlation between DHA and oxidative burst could reflect an indirect association with $18: 1 n-9$.

In general, changes in the fatty acid composition of all immune cells are related to the dose $\mathrm{s}^{(10,15)}$ and the length of 
intervention $^{(26)}$. The DHA content of the PBMC was significantly increased after 8 weeks of FO-supplementation as expected $^{(36)}$ and parallel changes have most likely also occurred in the neutrophils, as observed by Gibney \& Hunter ${ }^{(26)}$. The active NADPH oxidase-complex has been shown to be located in membrane domains, which are rich in saturated acyl groups, the so-called lipid rafts ${ }^{(20)}$. Lipid rafts are formed by segregation of cholesterol and sphingolipids from DHA-containing phospholipids ${ }^{(21)}$. It was therefore our baseline assumption, although we did not test this, that increased incorporation of DHA could lower the lipid raft area and have functional consequences equivalent to that of cholesterol extraction. Cholesterol depletion of HL-60 cells (a neutrophil-like cell line) has been shown to result in a dramatic reduction in NADPH oxidase activity ${ }^{(20)}$. Similar effects were observed in a number of other raft-associated proteins in Jurkat $\mathrm{T}$ cells and isolated human neutrophils $^{(35,37)}$. Furthermore, cholesterol depletion has been linked to a decrease in raft-associated protein trafficking ${ }^{(37)}$, and thus may affect the assembly of the NADPH oxidase complex. An ex vivo study of PUFA-supplemented Jurkat $\mathrm{T}$ cells showed a selective modification of unsaturation in the cytoplasmic layer of the detergent-resistant membrane domains (lipid rafts) and an associated inhibition of $\mathrm{T}$ cell signal transduction ${ }^{(35)}$. We did not determine whether the increased intake of $n-3$ LCPUFA had any effect on the microdomain structure and, to our knowledge, this has not been done in any previous studies. The observed effect of the capsule intervention corresponds poorly with this baseline assumption. The effect of the FO supplement on neutrophil function may therefore be explained by other mechanisms. Several effects of $n-3$ LCPUFA in other cell types (monocyte, B lymphocyte and $\mathrm{T}$ lymphocyte) are thought to be exerted via alterations in gene expression and transcription factors such as $\operatorname{PPAR}^{(38,39)}$. Gorjão et al. ${ }^{(29)}$ showed modification of seventy-seven genes in human lymphocytes after FO-supplementation, most of which were related to signalling pathways. According to the classical hypotheses, the immuno-suppressive effect of $n$-3 LCPUFA is thought to be caused by changes in eicosanoid production (e.g. modulation of the $\mathrm{LTB}_{4}$ levels $\left.{ }^{(40)}\right)$. However, blocking of eicosanoid production by a cyclo-oxygenase inhibitor (indomethacin) has been shown not to affect oxidative burst ${ }^{(22)}$.

In conclusion, the present study suggests an immuno-stimulating effect on oxidative burst after 8 weeks of FO-supplementation in healthy young men. The effect seems to be related to the cellular DHA content, but the mechanism remains to be determined.

\section{Acknowledgements}

This research was supported by a grant to L. L. from the Danish Medical Research Council (Grant No. 23-04-0050), and by a grant to H. S. H. from the Danish Medical Research Council (Grant No. 22-03-0374). Capsules, oils and fat spreads were kindly provided by Pharma Nord, Arla Foods amba, Aarhus United Denmark A/S and Unilever Denmark A/S Foods. The authors wish to thank all the young men who participated in the study. Furthermore, we thank co-workers Elin Skytte, Michael Seest and Anders Daniel for their help with data collection and Pia Madsen for performing the PBMC fatty acid analyses.

\section{References}

1. Anderson M \& Fritsche KL (2002) (n-3) Fatty acids and infectious disease resistance. $J$ Nutr 132, 3566-3576.

2. Kremer JM (2000) n-3 Fatty acid supplements in rheumatoid arthritis. Am J Clin Nutr 71, 349S-351S.

3. Calder PC (2006) n-3 Polyunsaturated fatty acids, inflammation, and inflammatory diseases. Am J Clin Nutr 83, 1505S-1519S.

4. Psota TL, Gebauer SK \& Kris-Etherton P (2006) Dietary omega-3 fatty acid intake and cardiovascular risk. Am J Cardiol 98, $3 \mathrm{i}-18 \mathrm{i}$.

5. Babior BM (2000) Phagocytes and oxidative stress. Am J Med 109, 33-44.

6. Ricevuti G (1997) Host tissue damage by phagocytes. Ann N Y Acad Sci 832, 426-448.

7. Sassen LM, Lamers JM, Sluiter W, Hartog JM, Dekkers DH, Hogendoorn A \& Verdouw PD (1993) Development and regression of atherosclerosis in pigs. Effects of $n-3$ fatty acids, their incorporation into plasma and aortic plaque lipids, and granulocyte function. Arterioscler Thromb 13, 651-660.

8. Fletcher MP \& Ziboh VA (1990) Effects of dietary supplementation with eicosapentaenoic acid or gamma-linolenic acid on neutrophil phospholipid fatty acid composition and activation responses. Inflammation 14, 585-597.

9. Thompson PJ, Misso NL, Passarelli M \& Phillips MJ (1991) The effect of eicosapentaenoic acid consumption on human neutrophil chemiluminescence. Lipids 26, 1223-1226.

10. Rees D, Miles EA, Banerjee T, Wells SJ, Roynette CE, Wahle KW \& Calder PC (2006) Dose-related effects of eicosapentaenoic acid on innate immune function in healthy humans: a comparison of young and older men. Am J Clin Nutr 83, 331-342.

11. Varming K, Schmidt EB, Svaneborg N, Moller JM, Lervang HH, Grunnet N, Jersild C \& Dyerberg J (1995) The effect of $n-3$ fatty acids on neutrophil chemiluminescence. Scand J Clin Lab Invest 55, 47-52.

12. Fisher M, Upchurch KS, Levine PH, Johnson MH, Vaudreuil CH, Natale A \& Hoogasian JJ (1986) Effects of dietary fish oil supplementation on polymorphonuclear leukocyte inflammatory potential. Inflammation 10, 387-392.

13. Guarini P, Bellavite P, Biasi D, Carletto A, Galvani S, Caramaschi P, Bambara LM \& Corrocher R (1998) Effects of dietary fish oil and soy phosphatidylcholine on neutrophil fatty acid composition, superoxide release, and adhesion. Inflammation 22, 381-391.

14. Healy DA, Wallace FA, Miles EA, Calder PC \& Newsholm P (2000) Effect of low-to-moderate amounts of dietary fish oil on neutrophil lipid composition and function. Lipids 35, $763-768$

15. Kew S, Banerjee T, Minihane AM, Finnegan YE, Muggli R, Albers R, Williams CM \& Calder PC (2003) Lack of effect of foods enriched with plant- or marine-derived $n$-3 fatty acids on human immune function. Am J Clin Nutr 77, 1287-1295.

16. Schmidt EB, Varming K, Moller JM, Bulow PI, Madsen P \& Dyerberg J (1996) No effect of a very low dose of $n-3$ fatty acids on monocyte function in healthy humans. Scand $J$ Clin Lab Invest 56, 87-92.

17. Thies F, Miles EA, Nebe-von-Caron G, Powell JR, Hurst TL, Newsholme EA \& Calder PC (2001) Influence of dietary supplementation with long-chain $n-3$ or $n-6$ polyunsaturated fatty acids on blood inflammatory cell populations and functions and on plasma soluble adhesion molecules in healthy adults. Lipids 36, 1183-1193.

18. Szekely A, Kitajka K, Panyi G, Marian T, Gaspar R \& Krasznai Z (2007) Nutrition and immune system: certain fatty acids differently modify membrane composition and consequently 
kinetics of KV1.3 channels of human peripheral lymphocytes. Immunobiology 212, 213-227.

19. Zhang X \& Young HA (2002) PPAR and immune system what do we know? Int Immunopharmacol 2, 1029-1044.

20. Vilhardt F \& van Deurs B (2004) The phagocyte NADPH oxidase depends on cholesterol-enriched membrane microdomains for assembly. EMBO $J \mathbf{2 3}, 739-748$.

21. Stillwell W \& Wassall SR (2003) Docosahexaenoic acid: membrane properties of a unique fatty acid. Chem Phys Lipids 126, 1-27.

22. Luostarinen R \& Saldeen T (1996) Dietary fish oil decreases superoxide generation by human neutrophils: relation to cyclooxygenase pathway and lysosomal enzyme release. Prostaglandins Leukot Essent Fatty Acids 55, 167-172.

23. Valentine RC \& Valentine DL (2004) Omega-3 fatty acids in cellular membranes: a unified concept. Prog Lipid Res 43, 383-402.

24. Kew S, Mesa MD, Tricon S, Buckley R, Minihane AM \& Yaqoob P (2004) Effects of oils rich in eicosapentaenoic and docosahexaenoic acids on immune cell composition and function in healthy humans. Am J Clin Nutr 79, 674-681.

25. Owen AJ, Peter-Przyborowska BA, Hoy AJ \& McLennan PL (2004) Dietary fish oil dose- and time-response effects on cardiac phospholipid fatty acid composition. Lipids 39, 955-961.

26. Gibney MJ \& Hunter B (1993) The effects of short- and longterm supplementation with fish oil on the incorporation of $n-3$ polyunsaturated fatty acids into cells of the immune system in healthy volunteers. Eur J Clin Nutr 47, 255-259.

27. Shao D, Segal AW \& Dekker LV (2003) Lipid rafts determine efficiency of NADPH oxidase activation in neutrophils. FEBS Lett 550, 101-106.

28. Damsgaard CT, Frokiaer H \& Lauritzen L (2007) The effects of fish oil and high or low linoleic acid intake on fatty acid composition of human peripheral blood mononuclear cells. $\mathrm{Br} J$ Nutr (In the Press).

29. Gorjao R, Verlengia R, Lima TM, et al. (2006) Effect of docosahexaenoic acid-rich fish oil supplementation on human leukocyte function. Clin Nutr 25, 923-938.

30. Geleijnse JM, Giltay EJ, Grobbee DE, Donders AR \& Kok FJ (2002) Blood pressure response to fish oil supplementation: metaregression analysis of randomized trials. J Hypertens $\mathbf{2 0}$, $1493-1499$.
31. Christensen JH, Christensen MS, Dyerberg J \& Schmidt EB (1999) Heart rate variability and fatty acid content of blood cell membranes: a dose-response study with $n-3$ fatty acids. Am J Clin Nutr 70, 331-337.

32. Pierre M, Husson MO, Le BR, et al. (2007) Omega-3 polyunsaturated fatty acids improve host response in chronic Pseudomonas aeruginosa lung infection in mice. Am J Physiol Lung Cell Mol Physiol 292, L1422-L1431.

33. Yaqoob P, Knapper JA, Webb DH, Williams CM, Newsholme EA \& Calder PC (1998) Effect of olive oil on immune function in middle-aged men. Am J Clin Nutr 67, 129-135.

34. Logan AC (2005) Omega-3 and depression research: hold the olive oil. Prostaglandins Leukot Essent Fatty Acids 72, 441.

35. Stulnig TM, Berger M, Sigmund T, Raederstorff D, Stockinger H \& Waldhausl W (1998) Polyunsaturated fatty acids inhibit T cell signal transduction by modification of detergent-insoluble membrane domains. J Cell Biol 143, 637-644.

36. Yaqoob P, Pala HS, Cortina-Borja M, Newsholme EA \& Calder PC (2000) Encapsulated fish oil enriched in alpha-tocopherol alters plasma phospholipid and mononuclear cell fatty acid compositions but not mononuclear cell functions. Eur J Clin Invest 30, 260-274

37. Kannan KB, Barlos D \& Hauser CJ (2007) Free cholesterol alters lipid raft structure and function regulating neutrophil $\mathrm{Ca}^{2+}$ entry and respiratory burst: correlations with calcium channel raft trafficking. J Immunol 178, 5253-5261.

38. Verlengia R, Gorjao R, Kanunfre CC, Bordin S, Martins De LT, Martins EF \& Curi R (2004) Comparative effects of eicosapentaenoic acid and docosahexaenoic acid on proliferation, cytokine production, and pleiotropic gene expression in Jurkat cells. J Nutr Biochem 15, 657-665.

39. Verlengia R, Gorjao R, Kanunfre CC, Bordin S, de Lima TM, Martins EF, Newsholme P \& Curi R (2004) Effects of EPA and DHA on proliferation, cytokine production, and gene expression in Raji cells. Lipids 39, 857-864.

40. Kelley DS, Taylor PC, Nelson GJ, Schmidt PC, Ferretti A, Erickson KL, Yu R, Chandra RK \& Mackey BE (1999) Docosahexaenoic acid ingestion inhibits natural killer cell activity and production of inflammatory mediators in young healthy men. Lipids 34, 317-324. 